\title{
Numerical simulations of slow magnetosonic standing waves in a straight solar coronal slab
}

\author{
R. Ogrodowczyk ${ }^{1}$ and K. Murawski ${ }^{2}$ \\ 1 Department of Computer Sciences, The State University in Chełm, ul. Pocztowa 54, 22-100 Chełm, Poland \\ e-mail: rogrodow@pwsz.chelm.pl \\ 2 Group of Astrophysics and Gravity Theory, UMCS, ul. Radziszewskiego 10, 20-031 Lublin, Poland
}

Received 19 January 2007 / Accepted 25 January 2007

\begin{abstract}
Aims. We consider a simple model of a straight solar coronal slab to explore excitation and attenuation of impulsively triggered slow magnetosonic standing waves.

Methods. The full set of ideal magnetohydrodynamic equations is solved numerically in the limit of a two-dimensional approximation. Results. The numerical results reveal that pulses, launched initially in gas pressure, trigger the fundamental slow mode and its first harmonic, depending on the spatial location of these pulses. These modes are excited over 3-5 wave periods and they are strongly attenuated over a similar time-scale.

Conclusions. As a result of the transition of the initial pulse to the modal structure through energy transfer into the ambient solar corona and into the photospheric regions, as well as of coupling with fast magnetosonic waves that spread energy over the whole physical system, these slow modes are excited faster and they are attenuated more efficiently than in the one-dimensional case.
\end{abstract}

Key words. magnetohydrodynamics (MHD) - methods: numerical

\section{Introduction}

It has been verified observationally that the solar corona consists of myriads of loops able to sustain various oscillations, which include slow magnetosonic waves (e.g., Nakariakov \& Verwichte 2005). There are reported observations of two entities of slow waves: (a) propagating waves, which are of very high quality and last for a number of periods (De Moortel et al. 2002a,b) and (b) standing waves, which are of low quality and are attenuated very quickly. EUV observations with SOHO/SUMER and TRACE have revealed that standing slow magnetosonic oscillations seem to be often triggered impulsively by micro- or subflares near a loop foot-point (Wang et al. 2002). These oscillations are quickly excited and are strongly attenuated over several wave periods (Wang et al. 2003).

These observational findings stimulated intensive theoretical investigations. In particular, Nakariakov et al. (2000) derived the modified Burgers equation, which takes into account nonlinearity, viscosity, thermal conductivity, stratification and structuring of plasma in the loop. They found that dissipation and stratification are the main factors that influence the wave evolution. In another study, Nakariakov et al. (2000) and Tsiklauri et al. (2004) have demonstrated that in a coronal loop thermal energy release that is realized through the appropriate source term in the energy equation efficiently excites the global mode. The considered one-dimensional model included the effects of gravitational stratification, heat conduction, radiative losses, external heat input and Braginskii bulk viscosity. Taroyan et al. (2006) followed Nakariakov et al. (2000) and Tsiklauri et al. (2004), and reached the conclusion that the global mode can also be efficiently excited. Selwa et al. (2005) clarified that pressure pulses located very close to the loop apex are able to excite the first harmonic. All other pulse locations give rise to the fundamental mode. They found, however, that in their one-dimensional model, 6-7 wave periods are required to set up a standing mode, in contrast to observations (Wang et al. 2005). Diáz \& Roberts (2006) discussed by analytical means standing slow waves in density structured coronal loops. They showed that for high density contrast between footpoints and apex, the modes of the structured loop are rather different from the unstructured case. The theory of slow waves was recently reviewed by Roberts (2006). An extensive review of propagating longitudinal intensity fluctuations, observed in coronal loops, was presented by De Moortel et al. (2002b).

It was proposed that slow waves can be attenuated by several mechanisms, such as wave leakage into the chromosphere (Van Doorsselaere et al. 2004), lateral wave leakage due to curvature of loops (Roberts 2000; Zaqarashvili et al. 2006), phase mixing (Nakariakov et al. 1999; Ofman \& Aschwanden 2002), resonant absorption (Ruderman \& Roberts 2002) and non-ideal magnetohydrodynamic effects (2000). In particular, Ofman \& Wang (2002) have found that thermal conduction leads to rapid damping of slow standing waves, with a less significant contribution from compressive viscosity. Ofman \& Aschwanden (2002) showed that a nonlinear steepening of slow waves leads to their enhanced dissipation. De Moortel et al. (2002b) concluded that thermal conduction can account for the observed damping times of propagating slow waves.

The aim of this paper is to generalize the study performed by Selwa et al. (2005) by excitation of slow magnetosonic waves in solar coronal loops in a two-dimensional slab. The considered model partly removes the main shortcoming of the work of Selwa et al. (2005), which takes into account slow magnetosonic waves only, neglecting the presence of fast magnetosonic 
and Alfvén waves in the system. As a consequence, their model excluded the possibility of slow wave generation either through a linear coupling process with fast waves or through nonlinear forces exerted by the Alfvén wave. While in the present paper the Alfvén waves are absent from the system, we found that the presence of fast magnetosonic waves is an important factor in the excitation and attenuation of standing slow waves.

This paper is organized as follows: the numerical model is described in the following part of the paper. Numerical results are presented in Sect. 3. This paper is concluded by a summary of the main results in Sect. 4.

\section{Numerical model}

We describe coronal plasma by the time-dependent ideal magnetohydrodynamic equations

$$
\begin{aligned}
& \frac{\partial \varrho}{\partial t}+\nabla \cdot(\varrho \boldsymbol{V})=0, \\
& \varrho \frac{\partial \boldsymbol{V}}{\partial t}+\varrho(\boldsymbol{V} \cdot \nabla) \boldsymbol{V}=-\nabla p+\frac{1}{\mu}(\nabla \times \boldsymbol{B}) \times \boldsymbol{B}, \\
& \frac{\partial p}{\partial t}+\nabla \cdot(p \boldsymbol{V})=(1-\gamma) p \nabla \cdot \boldsymbol{V}, \\
& \frac{\partial \boldsymbol{B}}{\partial t}=\nabla \times(\boldsymbol{V} \times \boldsymbol{B}), \\
& \nabla \cdot \boldsymbol{B}=0 .
\end{aligned}
$$

Here $\varrho$ is mass density, $\boldsymbol{V}$ flow velocity, $\boldsymbol{B}$ magnetic field, $p$ gas pressure, $\mu$ the magnetic permeability, and $\gamma=5 / 3$ is the adiabatic index.

\subsection{A slab equilibrium}

We limit our discussion to a two-dimensional magnetically structured atmosphere in which the plasma quantities are assumed to be independent of the spatial coordinate $y, \partial / \partial y=0$, and $V_{y}=B_{y}=0$. As a consequence of this assumption the Alfvén waves are removed from the system. Without any loss of generality, we set the initial, unperturbed (i.e. equilibrium) magnetic field to be directed parallel to the $z$-axis (Fig. 1). A coronal slab is approximated by plasma profiles in which the inhomogeneity occurs in the $x$-direction only. In particular, we consider a slab with a half-width $w$ and mass density $\varrho_{\mathrm{i}}=r \varrho_{\mathrm{e}}$, embedded in a magnetic environment with field strength $B_{\mathrm{e}}$ and mass density $\varrho_{\mathrm{e}}$. Here $r$ is the slab mass density contrast. We assume that $\varrho_{\mathrm{e}}$ jumps to the photospheric mass density value $\varrho_{\mathrm{ph}}=d \varrho_{\mathrm{e}}$ at the photospheric levels, with photospheric mass density contrast $d$. These photospheric levels are located on the left and right sides of the simulation region (Fig. 1). The Alfvén $c_{\mathrm{Ai}, \mathrm{e}}$ and sound $c_{\mathrm{si}, \mathrm{e}}$ speeds are defined as $c_{\mathrm{Ai}, \mathrm{e}}^{2}=B_{\mathrm{e}}^{2} /\left(\mu \varrho_{\mathrm{i}, \mathrm{e}}\right)$ and $c_{\mathrm{si}, \mathrm{e}}^{2}=\gamma p_{\mathrm{e}} / \varrho_{\mathrm{i}, \mathrm{e}}$, where the indices $i$ and $e$ correspond to the slab and ambient media, respectively. The plasma $\beta$ is expressed as $\beta=2 \mu p_{\mathrm{e}} / B_{\mathrm{e}}^{2}$ and as a result of $p_{\mathrm{e}}=$ const. and $B_{\mathrm{e}}=$ const. it is constant in the overall physical region. Numerical values for these parameters are presented in Table 1 . The ambient pressure $p_{\mathrm{e}}$ and magnetic field $B_{\mathrm{e}}$ are then described by the following formula:

$p_{\mathrm{e}}=\frac{1}{\gamma} c_{\mathrm{se}}^{2} \rho_{\mathrm{e}}, \quad B_{\mathrm{e}}=\sqrt{\frac{2 \mu}{\beta} p_{\mathrm{e}} .}$

The tube speed, $c_{\mathrm{T}}=c_{\mathrm{S}} c_{\mathrm{A}} / c_{\mathrm{f}}$, is displayed in Fig. 2 across the slab. Here the fast speed $c_{\mathrm{f}}$ is defined as $c_{\mathrm{f}}^{2}=c_{\mathrm{s}}^{2}+c_{\mathrm{A}}^{2}$, where

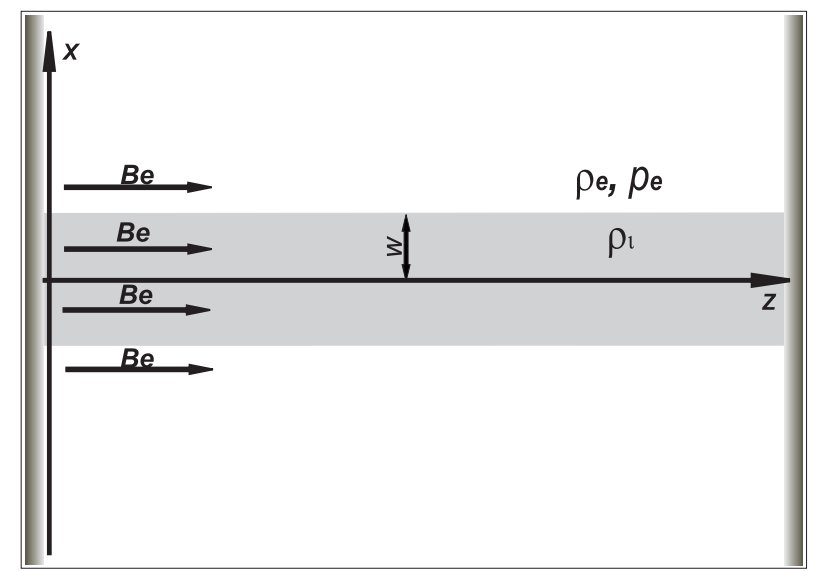

Fig. 1. Geometry of the model. The gray scale represents the mass density which jumps abruptly at the slab edges. Notice the dense photospheric regions that are located on the left and right sides of the simulation region.

Table 1. Equilibrium parameters.

\begin{tabular}{cc}
\hline \hline Quantity & Value \\
\hline$c_{\mathrm{Ae}}$ & $1 \mathrm{Mm} \mathrm{s}^{-1}$ \\
$c_{\mathrm{se}}$ & $0.3 \mathrm{Mm} \mathrm{s}^{-1}$ \\
$\beta$ & 0.108 \\
$\varrho_{\mathrm{e}}$ & $10^{-15} \mathrm{~kg} \mathrm{~m}^{-3}$ \\
$r=\varrho_{\mathrm{i}} / \varrho_{\mathrm{e}}$ & 3 \\
$d=\varrho_{\mathrm{ph}} / \varrho_{\mathrm{e}}$ & $10^{4}$ \\
$w$ & $1 \mathrm{Mm}$ \\
\hline
\end{tabular}

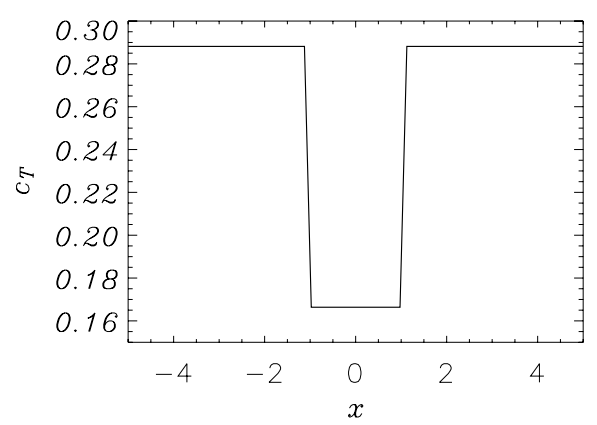

Fig. 2. The tube speed, $c_{\mathrm{T}}$, across the slab.

$c_{\mathrm{s}}=c_{\mathrm{S}}(x)$ and $c_{\mathrm{A}}=c_{\mathrm{A}}(x)$ are respectively the local sound and Alfvén speeds.

\subsection{Perturbations}

Perturbations of the slab equilibrium can be triggered in numerous ways. As we are interested in impulsively excited waves, we launch a pulse in gas pressure at $t=0$. We assume that this pulse has the following form:

$p(x, z, t=0)= \begin{cases}p_{\mathrm{e}}\left[1+A_{\mathrm{p}} \mathrm{e}^{\left.-\left(\frac{z-z_{0}}{a}\right)^{2}\right]}\right], & |x| \leq w, \\ 0, & |x|>w .\end{cases}$

Here $A_{\mathrm{p}}$ denotes the relative amplitude of the initial pulse, $z_{0}$ its spatial position, and $a$ its width. In our studies we choose $A_{\mathrm{p}}=$ 0.5 and $a=L / 40=1.25 \mathrm{Mm}$, where $L$ is the length of the slab, which we set as $L=50 \mathrm{Mm}$. These parameters correspond to a relatively short coronal loop. 
It is noteworthy that the pulse, given by Eq. (7), triggers fast and slow magnetosonic waves which are simultaneously present in the system as a result of $\beta$ different from zero. In the limit of small amplitude waves, fast and slow waves are governed by linearized equations

$\left[\varrho_{0}\left(\frac{\partial^{2}}{\partial t^{2}}-c_{\mathrm{A}}^{2} \frac{\partial^{2}}{\partial z^{2}}\right)-\frac{\partial}{\partial x} \varrho_{0} c_{\mathrm{f}}^{2} \frac{\partial}{\partial x}\right] V_{x}=\frac{\partial}{\partial x} \varrho_{0} c_{\mathrm{s}}^{2} \frac{\partial}{\partial z} V_{z}$,

$\left(\frac{\partial^{2}}{\partial t^{2}}-c_{\mathrm{s}}^{2} \frac{\partial^{2}}{\partial z^{2}}\right) V_{z}=c_{\mathrm{s}}^{2} \frac{\partial^{2}}{\partial x \partial z} V_{x}$

These equations describe the longitudinal $V_{z}$ and transverse $V_{x}$ motions. In general, both velocity components, $V_{x}$ and $V_{z}$ are present in both fast and slow magnetosonic waves. However, when the plasma beta tends to zero, the longitudinal motions are associated with the slow modes only, and the transverse motions with the fast modes only. From Eq. (2) it follows that $V_{x}\left(V_{z}\right)$ is driven by $-\frac{\partial p}{\partial x}\left(-\frac{\partial p}{\partial z}\right)$ which initially attains high values at the slab edges (inside the slab) as a result of the initial pressure pulse of Eq. (7). As a consequence of that the initial pulse triggers fast (slow) waves at the slab edges (inside the slab).

Equations (8) and (9) are only shown for illustration and they are not solved numerically. Instead, the full set of Eqs. (1)-(5) is treated numerically. The details of this treatment are presented in the following section.

\section{Numerical results}

In our approach, we adopt the numerical code Athena, which was developed by Gardiner et al. (2005). In this code a higherorder Godunov method is implemented to solve the timedependent, ideal magnetohydrodynamic equations. The algorithm is single step, second-order accurate in space and time and it is based on the piecewise parabolic method (e.g., Woodward \& Collela 1984) that is supported by the constrained transport scheme to keep the divergence-free constraint on the magnetic field (e.g., Murawski 2002).

We solved numerically Eqs. (1)-(5) with the use of a Eulerian grid with the $x$ - and $z$-dimensions $(-16 l, 16 l) \times$ $(-10 l, 60 l)$, where $l$ is a spatial unit, chosen as $l=1 \mathrm{Mm}$. This box is covered by a uniform grid of $200 \times 300$ numerical cells. We performed grid convergence studies which are based on grid refinement to show that the numerical results are not affected by insufficient spatial resolution. We apply open boundary conditions with zero-gradient extrapolation of all plasma variables, allowing a wave signal to freely leave the simulation region. Spatial coordinates, velocities and time are expressed in units of $l, c_{\mathrm{Ae}}$ and seconds, respectively.

\subsection{The fundamental mode}

Here, we consider an initial pulse that is launched at $z_{0}=L / 4$. Figure 3 shows spatial profiles of $V_{z}$ at two different times (top panels) and the corresponding slices of $V_{z}$, drawn along the line $x=0$ (middle panels) and $z=L / 2$ (bottom panels). These profiles exhibit spatial profiles of $V_{z}$ which are approximately in anti-phases, corresponding to the fundamental mode. The wave profiles attain large gradients across the slab. These gradients are a consequence of large variations of tube speed at the slab edges (Fig. 2). The slow wave propagates with a phase speed which is close to $c_{\mathrm{T}}$. As $c_{\mathrm{T}}$ is higher in the ambient medium than within the slab a non-zero gradient of $V_{z}(x)$ results in the $x$-direction.

Slow waves are not confined to the slab only; they are also present in the ambient medium too. It is discernible from the
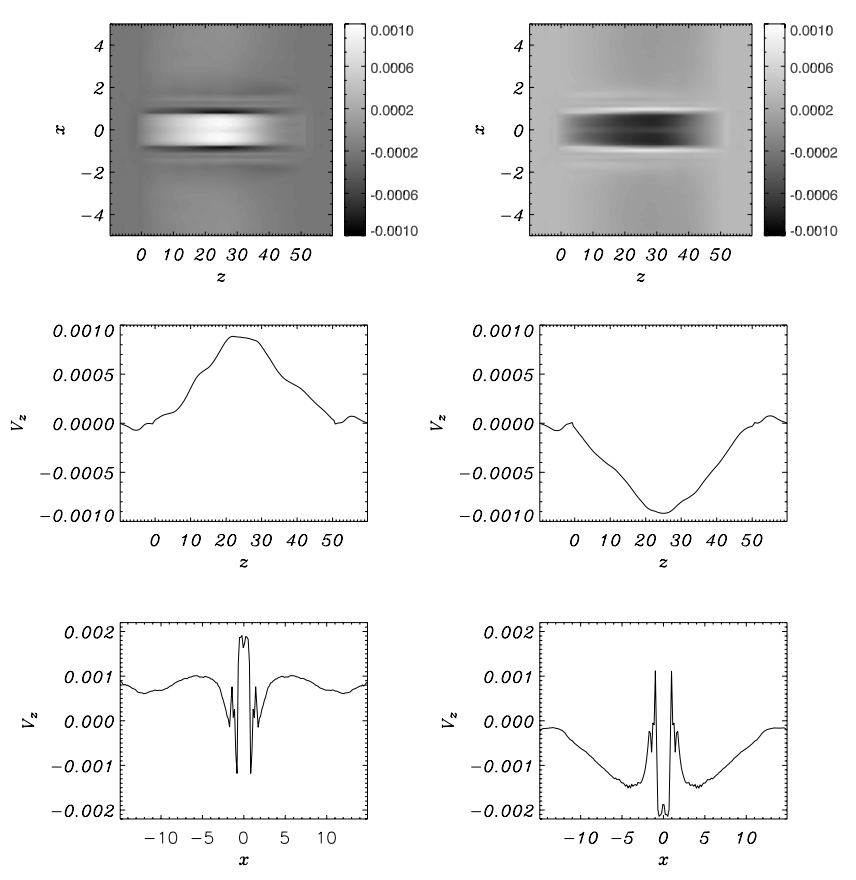

Fig. 3. Top panels: spatial profiles of the parallel component of velocity $V_{z}$ at $t \simeq 6.5 P_{1}$ (left panels) and $t \simeq 6.9 P_{1}$ (right panels) for the initial pulse position $z_{0}=L / 2$. Here $P_{1}$ is the wave period of the fundamental standing slow wave, defined in Eq. (11). The corresponding slices of $V_{z}$ along $x=0(z=L / 2)$ are shown in the middle (bottom) panels.

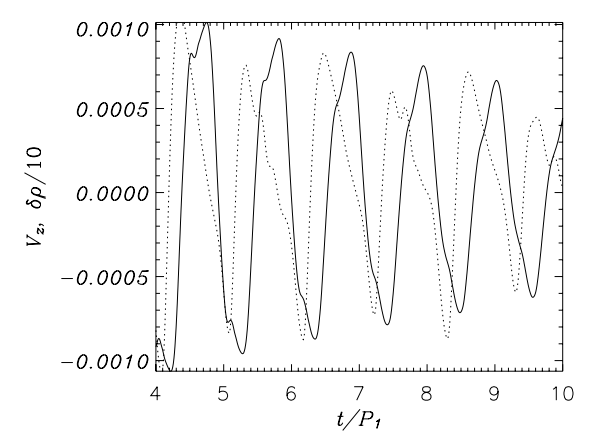

Fig. 4. The slices of time-signatures of $V_{z}$ (solid line) and $\delta \varrho$ (dotted line) along $x=0$ evaluated within the slab at $\left(z_{\mathrm{d}}=L / 4\right)$ for the initial pulse position $z_{0}=L / 4$. Note a quarter wave-period phase-shift between $\delta \varrho$ and $V_{z}$.

bottom panels of Fig. 3 that the plasma in the ambient medium exhibits oscillations. The slow modes are present outside the slab, because of the evanescent solution. The departure from that evanescent behavior can be explained by the slow wave transition to the exact modal solution.

Slow waves produce characteristic time-signatures which are made by collecting a wave signal at a given spatial location. Figure 4 displays the slices of time-signatures of $V_{z}$ (solid line) and $\delta \varrho$ (dotted line) collected at $(x=0, z=L / 4)$. These timesignatures are reminiscent of attenuated oscillations which may result from the transition behaviour of the perturbations that evolve towards the exact solution. Note a phase-shift $\delta \phi$ between mass density $\delta \varrho$ and $V_{z}$ (bottom panel). This phase-shift can be used to determine the presence of slow standing waves in 


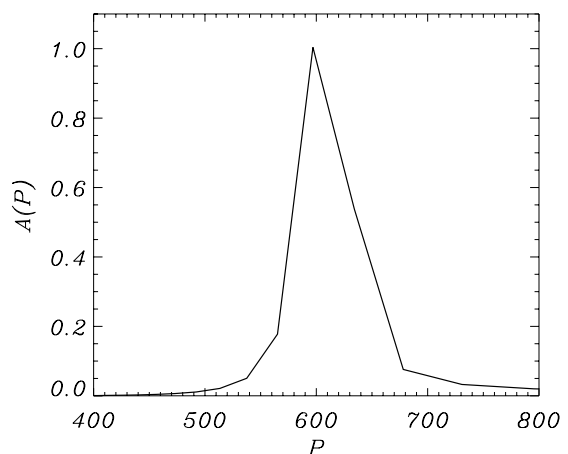

Fig. 5. Fourier spectrum of $V_{z}(x=0, z=L / 4, t)$ of the wave signals of Fig. 4. The Fourier analysis reveals a wave period of $P_{1} \cong 600 \mathrm{~s}$. The amplitude of the power spectrum $A(P)$ is normalized to 1 .

a physical system; $\delta \phi$ is equal to a quarter of a wave period for a pure slow standing wave (e.g., Nakariakov \& Verwichte 2005). We use an excitation criterion, according to which a standing wave is present in the system if $\delta \phi / P_{\mathrm{n}}$ departs by $20 \%$ from $1 / 4$ (Selwa et al. 2005),

$\frac{1}{4} \cdot 80 \% \leq \delta \phi / P_{\mathrm{n}} \leq \frac{1}{4} \cdot 120 \%$.

Here

$P_{\mathrm{n}}=\frac{2 L_{\mathrm{eff}}}{n c_{\mathrm{Ti}}}, n=1,2, \ldots$

is the wave period of a standing slow wave with $n$ denoting the standing wave number, $c_{\mathrm{Ti}}=0.1716 \mathrm{Mms}^{-1}$ is the tube speed inside the slab and $L_{\text {eff }}$ denotes the effective loop length. This criterion is fulfilled for $t>t_{\mathrm{ex}}$, where $t_{\mathrm{ex}}$ is called the excitation time. We found that the fundamental standing wave is excited at $t_{\mathrm{ex}} \approx 4 P_{1}$ (Fig. 4 ).

Figure 5 displays the Fourier spectrum of $V_{z}$, evaluated at ( $x=0, z=L / 4, t)$. This spectrum reveals the presence of the fundamental mode $n=1$ with wave period $P_{1} \cong 600 \mathrm{~s}$. From Eq. (11) we evaluate $L_{\text {eff }}=51.5 \mathrm{Mm}$ which is close to the slab length $L=50 \mathrm{Mm}$.

To estimate the attenuation time, $\tau$, we adopt the analytical formula:

$V_{z}(x=0, z=L / 4, t)=V_{z 0} \exp \left(-\frac{t-t_{\mathrm{ex}}}{\tau}\right)$,

and fit it into the time-signatures of $V_{z}$ from Fig. 4. Here $V_{z 0}$ denotes the amplitude of the parallel component of velocity, $V_{z}$, at time $t_{\text {ex }}$. We found that $\tau \cong 3.82 P_{1}$ for the fundamental standing wave.

It is noteworthy that both the excitation and attenuation times are smaller than in the 1d model of Selwa et al. (2005), who found that $t_{\mathrm{ex}} \geq 4 P_{1}$ and $\tau \approx 6.1 P_{1}$ (for comparable photospheric density contrast). We infer that the reduction in the attenuation time results from leakage of slow wave energy into the photospheric layers. As a consequence of this leakage, slow waves are more efficiently attenuated than in the $1 \mathrm{~d}$ case.

The longitudinal leakage can be enhanced by the divergence of the loop flux tube, which reduces the decay time. This effect is not discussed in this paper, however. As a result, the estimated time of the photospheric leakage cannot be directly applied to the interpretation of observations. Additionally, the sound speed should vary from $0.3 \mathrm{Mms}^{-1}$ in the solar corona to $0.01 \mathrm{Mm}^{-1}$ within the photospheric regions, while we implemented $c_{\mathrm{s}}=$ $0.3 \mathrm{Mms}^{-1}$ in the solar corona and $c_{\mathrm{s}}=0.003 \mathrm{Mms}^{-1}$ in the photosphere.
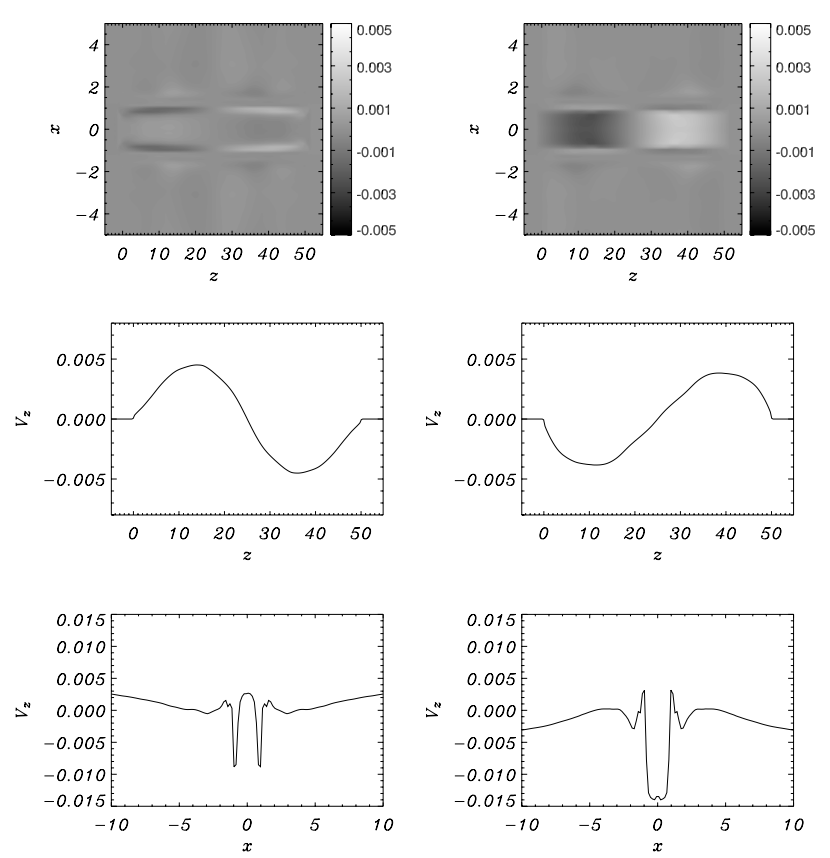

Fig. 6. Top panels: spatial profiles of the parallel component of velocity $V_{z}$ at $t \simeq 6.8 P_{2}$ (left panels) and $V_{z}$ at $t \simeq 7.3 P_{2}$ (right panels) for the initial pulse position $z_{0}=L / 2$. The corresponding slices of $V_{z}$ along $x=0(z=L / 4)$ are shown in the middle (bottom) panels.

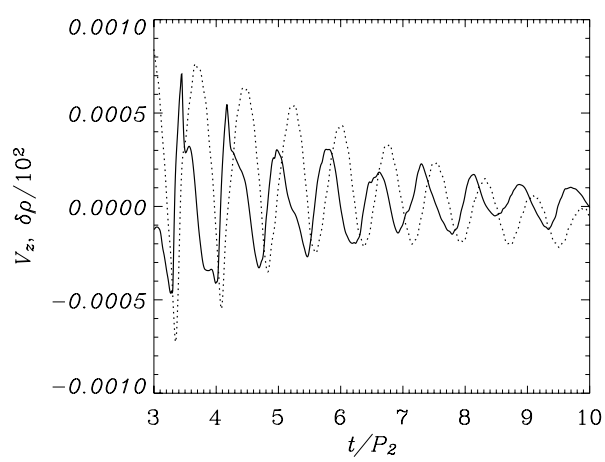

Fig. 7. Time-signatures of $V_{z}$ (solid line) and $\delta \varrho$ (dotted line), collected at $x_{\mathrm{d}}=0, z_{\mathrm{d}}=L / 4$ for $z_{0}=L / 2$.

\subsection{The first harmonic mode}

In this part of the paper we consider the case of the initial pulse that is launched at $z_{0}=L / 2$. Figure 6 illustrates spatial profiles of the parallel component of velocity $V_{z}$ and its slices, drawn along $x=0$ and $z=L / 4$ at two different times. For this pulse position we obtain the first harmonic mode, $n=2$ which confirms the findings of Selwa et al. (2005).

Figure 7 shows time-signatures of parallel velocity $V_{z}$ and $\delta \varrho$, evaluated at $(x=0, z=L / 4)$. Note that $V_{z}$ and $\delta \varrho$ exhibit a quarter wave-period phase-shift. The Fourier spectrum (Fig. 8) reveals the wave period $P_{2}=294.1 \mathrm{~s}$ of the first harmonic $(n=2)$ of the slow standing wave, which is close to the analytical value that is obtained from Eq. (11), $P_{2} \cong 289 \mathrm{~s}$. According to the excitation (attenuation) criterion of Eq. (10) (Eq. (12)), this mode is excited (attenuated) at $t_{\mathrm{ex}} \simeq 2.9 P_{2}\left(\tau \simeq 4.05 P_{2}\right)$. 


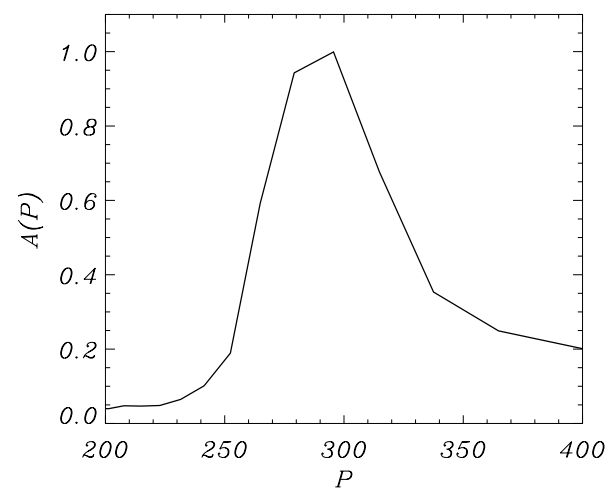

Fig. 8. Fourier spectrum of $V_{z}(x=0, z=L / 4, t)$ of the wave signals of Fig. 7. Fourier analysis leads to wave period $P_{2}=294.1 \mathrm{~s}$.
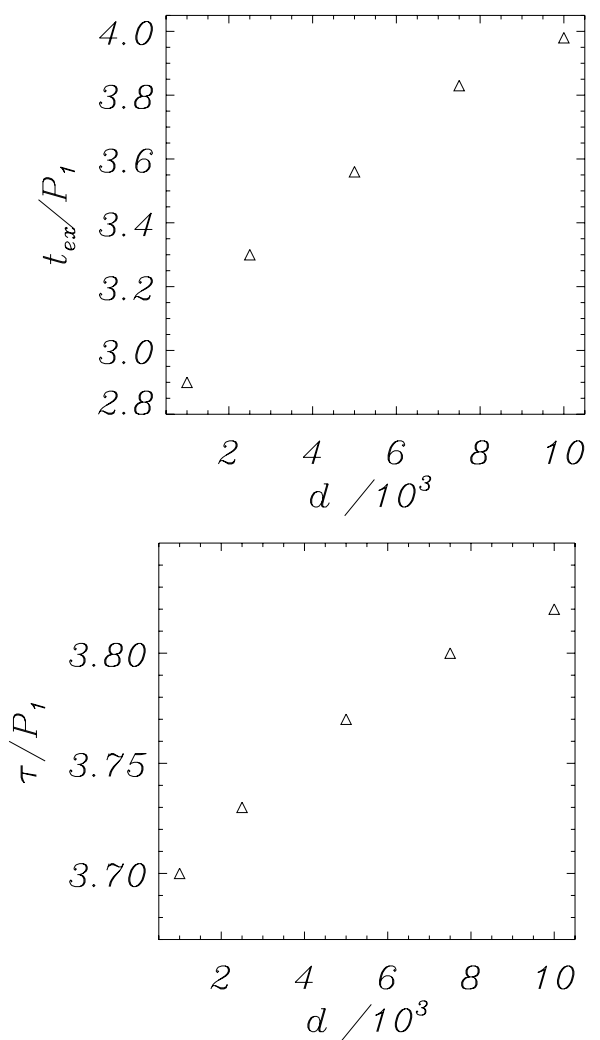

Fig. 9. Normalized standing slow wave excitation time $t_{\mathrm{ex}} / P_{1}$ (top panel) and attenuation time $\tau / P_{1}$ (bottom panel) vs. photospheric mass density contrast $d=\varrho_{\mathrm{ph}} / \varrho_{\mathrm{e}}$ for $z_{0}=L / 4$.

\subsection{Attenuation of slow waves}

We discuss attenuation of slow waves as a result of energy leakage into the photospheric regions. Figure 9 shows that both $t_{\mathrm{ex}} / P_{1}$ and $\tau / P_{1}$ grow with photospheric mass density contrast $d$. As a consequence of that we infer that energy leakage to the photospheric layers is a significant factor that is responsible for wave attenuation.

We present results for various values of plasma $\beta$. We change the value of $\beta$ by varying the value of the sound speed $c_{\mathrm{se}}$ in the ambient medium. We set and hold fixed $d=10^{4}$. As we already mentioned for a low value of plasma $\beta$ the role in fast waves on excitation and attenuation of slow waves is small. Such approach allows us to determine the influence of the coupling between fast and slow waves on the excitation and attenuation times of slow
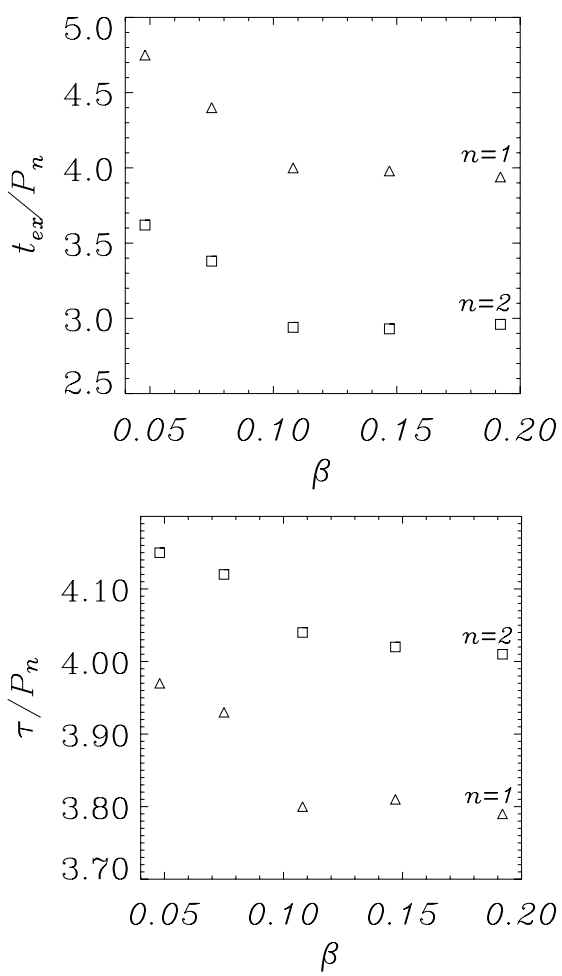

Fig. 10. Normalized standing slow wave excitation time $t_{\mathrm{ex}} / P_{\mathrm{n}}$ (top panel) and attenuation time $\tau / P_{\mathrm{n}}$ (bottom panel) vs. plasma $\beta$ for $z_{0}=L / 2$ (squares) and $z_{0}=L / 4$ (triangles). Here, $n=1,2$ denotes a mode number.

standing waves. This coupling is obviously stronger for a larger value of plasma $\beta$.

The top panel of Fig. 10 illustrates normalized standing wave excitation time $t_{\mathrm{ex}} / P_{\mathrm{n}}$ vs. plasma $\beta$ (top panel). As $t_{\mathrm{ex}} / P_{\mathrm{n}}$ declines with $\beta$ we infer that a stronger coupling between parallel $\left(V_{z}\right)$ and perpendicular $\left(V_{x}\right)$ components of velocity (see Eqs. (8), (9)) results in a shorter excitation time. As a consequence, slow standing waves are excited faster for larger values of $\beta$, for which the coupling between fast and slow waves is stronger. This declining trend in $t_{\mathrm{ex}} / P_{\mathrm{n}}$ results from fast magnetosonic waves which propagate faster than slow magnetosonic waves and spread their energy over the whole simulation region. Slow waves are driven by fast waves in the overall region and as a result, slow standing waves are sustained faster than in the case of a low value of $\beta$, when the coupling is weak.

It is noteworthy that the mode $n=2$ (squares) is excited more efficiently than the mode $n=1$ (triangles). This behavior results from symmetry reasons as the mode $n=2$ is triggered by the pulse launched from the slab center, $z_{0}=L / 2$. As a consequence, it takes less time to spread the initial pulse energy over the whole slab than in the case of $z_{0}=L / 4$ which corresponds to the mode $n=1$.

Figure 10 (bottom panel) displays the normalized attenuation time $\tau / P_{\mathrm{n}}$ as a function of plasma $\beta$. To estimate $\tau$ we adopt the analytical formula described by Eq. (12). We find that values of $\tau / P_{\mathrm{n}}$ are in the range $3.79<\tau / P_{1}<3.97\left(4.0<\tau / P_{2}<4.15\right)$ for the fundamental (the first harmonic) standing wave. The normalized attenuation time $\tau / P_{\mathrm{n}}$ declines with $\beta$. A stronger coupling between $V_{z}$ and $V_{x}$ results in a smaller value of $\tau / P_{\mathrm{n}}$. As a consequence of this coupling, slow waves transfer their energy into fast waves more efficiently and they become more strongly attenuated for a larger value of $\beta$. As $\tau / P_{1}<\tau / P_{2}$ we infer that the mode $n=1$ (triangles) is attenuated more efficiently than the 
mode $n=2$ (squares). Our results are in general agreement with the findings of Zaqarashvili et al. (2006) who discussed standing slow magnetosonic waves in a curved coronal loop.

\section{Summary}

We developed a two-dimensional model of a coronal slab to discuss by numerical means standing slow magnetosonic waves. These waves are triggered by a pressure pulse that is initially launched inside the slab. Such a pulse excites magnetosonic waves among which slow waves are dominant. Depending on the position of this pulse either fundamental or first harmonic slow modes are generated. Our numerical results show that slow modes are excited and attenuated faster than in the onedimensional case (Selwa et al. 2005). We conclude that leakage of slow waves into the photospheric regions and energy transfer during the initial transient stage into the ambient medium is responsible for this scenario. The observed attenuation of the oscillations is not associated with any non-ideal effect that results in dissipation of eigen modes, but instead with the transition of the impulsive excitation to the eigen mode solution.

Acknowledgements. R.O. and K.M. thank Prof. Sami Solanki and Dr. Valery Nakariakov for comments that helped to improve this paper and to Dr. Temury Zaqarashvili for stimulating discussions. K.M. thanks Tom Gardiner for discussions on several numerical issues associated with the code Athena. The magnetohydrodynamics code used in this study was developed at Princeton University by Tom Gardiner, Jim Stone, Peter Teuben and John Hawley with the support of the NSF Information Technology Research (ITR) program.

\section{References}

De Moortel, T., Hood, A. W., Ireland, J., \& Walsh, R. W. 2002a, Sol. Phys., 209, 89

De Moortel, I., Hood, A. W., \& Ireland, J. 2002b, A\&A, 381, 311

Diáz, A. J., \& Roberts, B. 2006, A\&A, 458, 975

Gardiner, T. A., \& Stone, J. M. 2005, J. Comput. Phys., 205, 509

Murawski, K. 2002, Analytical and numerical methods for wave propagation in Fluid Media (Singapore: World Scientific)

Nakariakov, V. M., Ofman, L., DeLuca, E. E., Roberts, B., \& Davila, J. M. 1999, Science, 285,862

Nakariakov, V. M., Verwichte, E., Berghmans, D., \& Robbrecht, E. 2000, A\&A, 362,1151

Nakariakov, V. M., \& Verwichte, E. 2005, Liv. Rev. Sol. Phys., 2, 3

Nakariakov, V. M., Tsiklauri, D., Kelly, A., Arber, T. D., \& Aschwanden, M. J. 2004, A\&A, 414, L25

Ofman, L., \& Aschwanden, M. 2002, ApJ, 576, L153

Ofman, L., \& Wang, T. J. 2002, ApJ, 580, L85

Roberts, B. 2000, Sol. Phys., 193, 139

Roberts, B. 2006, R. Soc. London Trans. Ser. A, 364, 447

Ruderman, M. S., \& Roberts, B. 2002, ApJ, 577, 475

Selwa, M., Murawski, K., \& Solanki, S. K. 2005, A\&A, 436, 701

Taroyan, Y., Erdélyi, R., Doyle, J. G., \& Bradshaw, S. J. 2006, A\&A, 438, 713

Tsiklauri, D., Nakariakov, V. M., Arber, T. D., \& Aschwanden, M. J. 2004, A\&A, 422,351

Van Doorsselaere, T., Debosscher, A., Andries, J., \& Poedts, S. 2004, A\&A, 424, 1065

Wang, T. J., Solanki, S. K., Curdt, W., Innes, D. E., \& Dammasch, I. E. 2002, ApJ, 574, L101

Wang, T. J., Solanki, S. K., Innes, D. E., Curdt, W., \& Marsch, E. 2003, A\&A, 402, L17

Wang, T. J., Solanki, S. K., Innes, D. E., \& Curdt, W. 2005, A\&A, 435, 753

Woodward, P., \& Collela, P. 1984, J. Comput. Phys., 54, 115

Zaqarashvili, T. V., Oliver, R., \& Ballester, J. L. 2006, A\&A, submitted 